many were those of long-legged wading birds. But the most interesting are those of the Mammoth and the problematical so-called human tracks. About the Mammoth tracks there can be no doubt. Some of these were uncovered by blasting in my presence; round basin-shaped impressions, 5 inches deep and 22 inches across, and occurring in regular alternating series, the hind-foot tracking almost perfectly with the fore-foot. The nature of the so-called human tracks, however, is far more doubtful. These occur in several regular alternating series of 15-20. In size they are $18-20$ inches long, and 8 inches wide. In shape they are many of them far more curved than the human track, especially in soft mud. The stride is $2 \frac{1}{2}$ to 3 feet, and even more. The outward turn of the track is in many cases greater than in human tracks, especially in soft mud. But the most remarkable thing about them on the human theory is the straddle, i.e. the distance between the right and left series. This I found to be 18 and even 19 inches, which was fully as great as that of the mammoth tracks. This is probably the greatest objection to the human theory. On the other hand, the great objection to the quadrupedal theory is the apparent singleness of the tracks, and the absence of claw-marks. But it must be remembered that the tracks are deep, and the outlines somewhat obscure, and also that the mammoth tracks, on account of tracking of hind with fore-foot, are in most cases, though not always, single.

After careful examination for several days, the conclusion I came to was that the tracks were probably made by a large plantigrade quadruped, most likely a gigantic ground-sloth, such as the Mylodon, which is found in the Quaternary, oi the Morothenium, which is found in the upper Plocene of Nevada. The apparent singleness, the singular shape, and the large outward turn of the tracks I attribute to the imperfect tracking of hind and fore-foot on the same side, while the absence of clawmarks was the result of the clogging of the feet with mud.

This view seems to me most probable, ${ }^{1}$ but many who have seen the tracks think them human, and I freely admit that there is abundant room for honest difference of opinion. On any theory the tracks are well worthy of scientific attention.

Berkeley, California, May I2

JOSEPH LE CONTE

\section{Cloudiness of Aquarium}

CAN you tell me the reason why the water in my fresh water aquarium will not remain clear, but becomes cloudy throughout in a few days after filling.

The aquarium in question holds about twelve gallons of water. It stands in a window facing north. I have in the water two or three water-plants, among them a water-aloe. At the bottom are small gravel stones, which have been thoroughly washed before using. Floating on the surface for the benefit of a few newts is a piece of virgin cork, on which is placed some carpet moss. I had a dozen minnows and four newts to begin with, but nine of the minnows and two of the newts have died, manifestly from the fouling of the water.

The framework of the aquarium is iron, with a slate floor. The glass sides are fixed with red lead. There is a copper tube for overflow purposes, which was inserted when a fountain was used in the centre. This has now been removed and the water is stagnant.

It is now some years since I have kept an aquarium, and cannot divine the reason for the above-mentioned cloudiness of the water. I shall be much obliged if you or some of your correspondents will help me.

$\mathrm{X}$.

May 9

So far as I can judge from "X's" description, the cloudiness of the water in his aquarium is due to the abnormal development of some unicellular algal (Palmellaceæ) or to the prolific spore-production within it of one of the filamentous forms (Confervacer). This may be obviated by screening the back of the tank from the access of light. Possibly "X" may find on examination that the cistern whence he obtains his supply has been left uncovered, and that the intruding algal has established itself and entered upon the reproductive process in that position. In that case he should either isolate the water he requires in a dark place for a week or so, when the spores will die, or obtain his supply from a purer source. An investigation with a high po ver of the microscope of the turbid water complained of will

${ }^{x}$ Views similar to my own have recently been expressed by Prof. Marsh and by $\mathrm{G}$. K. Gilbert. speedily determine whether the explanation here suggested is the correct one. By way of illustration, I may mention that the water of the ornamental pond in the centre of the Horticultural Gardens, supplied clear and bright shortly before the opening of the Fisheries Exhibition, had assumed within a few days and still retains the colour and consistency of green-pea soup through the rapid development, under the action of light, of a unicellular cryptogam in the manner above described.

W. SAVille KeNT

\section{Singing, Speaking, and Stammering}

REFERRING to the letters in NATURE (vol. xxvii. p. 580) on my classification of vowel sounds, allow me to explain :-

The classification given in the "Principles of Elocution" (4th ed., 1878) was retained from the earlier editions of that work, because of the difficulty, or impossibility, of exhibiting the complete vowel system of visible speech without V.S. symbols. For the purposes of the book on Elocution, the latter were not required; but a note (on p. 36) immediately preceding the "General Vowel Scheme" explains the basis of the complete classification developed in visible speech.

As you have given an abstract of my classification, quoted by Dr. Stone from " Principles of Elocution," I shall be glad if you will show your readers the following abstract of the visible speech classification :-

\section{Classification of Vowels in Visible Speech}

Nine Lingual positions yield 9 Primary vowels ...

Each Primary vowel yields a "Wide" variety by faucal expansion $=9$ Wide vowels

Each Lingual vowel yields a "Round" variety by $\}=18$ Labio-lingual labial contraction ........

Each Normal vowel yields a possible variety by higher, lower, broader, or narrower formation $=36+144=a$ total of 180 vowels.

The mutual relations of the different sounds may be exhibited in this way :-

\begin{tabular}{|c|c|c|c|c|c|c|}
\hline & \multicolumn{6}{|c|}{ LINGUAL. } \\
\hline & \multicolumn{3}{|c|}{ Primary. } & \multicolumn{3}{|c|}{ Wide. } \\
\hline & Back. & Mixed. & Front. & Back. & Mixed. & Front. \\
\hline High & 7 & 4 & I & 7 & 4 & I \\
\hline Mid & 8 & 5 & 2 & 8 & 5 & 2 \\
\hline Low & 9 & 6 & 3 & 9 & 6 & 3 \\
\hline
\end{tabular}

\begin{tabular}{|c|c|c|c|c|c|c|}
\hline & \multicolumn{6}{|c|}{ LABIO-LINGUAL. } \\
\hline & \multicolumn{3}{|c|}{ Primary. } & \multicolumn{3}{|c|}{ Wide. } \\
\hline & Back. & Mixed. & Front. & Back. & Mixed. & Front. \\
\hline High & 7 & 4 & I & 7 & 4 & I \\
\hline Mid & 8 & 5 & 2 & 8 & 5 & 2 \\
\hline Low & 9 & 6 & 3 & 9 & 6 & 3 \\
\hline
\end{tabular}

In this arrangement, each No. 1, No. 2, No, 3, \&c., in the four sets is formed from one and the same lingual position. These relations are plainly exhibited in the symbuls of visible speech. They cannot be shown by ordinary letters, but the use of numbers, as above, may make the arrangement clear to those who are not acquainted with visible speech.

Washington, D.C., May 12 Alex. Melville Beli

\section{On the Cold in March, and Absence of Sunspots}

I wAS travelling when Dr. Woeikof's letter appeared in NATURE (vol. xxviii. p. 53), and could not sooner reply to his criticisms on my communication (vol. xxvii. p. 551), "Unprecedented Cold in the Riviera-Absence of sunspots." Let me first remark that I do not go so far as to "ascribe (as Dr. Woeikof says that $I$ do) the great cold of March, 1883, at the 